November 2007

\title{
Effect of Charged Scalar Loops on Photonic Decays of a Fermiophobic Higgs
}

\author{
A.G. Akeroyd ${ }^{a, b}$, Marco A. Díaz $^{c}$, Maximiliano A. Rivera $^{c}$ \\ a: Department of Physics, National Cheng Kung University, Tainan 701, Taiwan \\ b: National Center for Theoretical Sciences, Taiwan \\ c: Departamento de Física, Universidad Católica de Chile, \\ Avenida Vicuña Mackenna 4860, Santiago, Chile
}

\begin{abstract}
Higgs bosons with very suppressed couplings to fermions ("Fermiophobic Higgs bosons", $\left.h_{f}\right)$ can decay to two photons $(\gamma \gamma)$ with a branching ratio significantly larger than that expected for the Standard Model Higgs boson for $m_{h_{f}}<150 \mathrm{GeV}$. Such a particle would give a clear signal at the LHC and can arise in the Two Higgs Doublet Model (type I) in which $h_{f} \rightarrow \gamma \gamma$ is mediated by $W^{ \pm}$and charged Higgs boson $\left(H^{ \pm}\right)$loops. We show that the $H^{ \pm}$loops can cause both constructive and destructive contributions with a magnitude considerably larger than the anticipated precision in the measurement of the photonic decay channel at future hadron and lepton colliders.
\end{abstract}




\section{Introduction}

Neutral Higgs bosons with very suppressed couplings to fermions - "fermiophobic Higgs bosons" $\left(h_{f}\right)[1]-$ may arise in specific versions of the Two Higgs Doublet Model (2HDM) [2, 3] or in models with Higgs triplets [4]. Such a $h_{f}$ would decay dominantly to two photons, $h_{f} \rightarrow \gamma \gamma$, for $m_{h_{f}} \lesssim 95 \mathrm{GeV}$ or to two massive gauge bosons, $h_{f} \rightarrow V V^{(*)}$, $\left(V=W^{ \pm}, Z\right)$ for $m_{h_{f}} \gtrsim 95 \mathrm{GeV}[5,6]$. The large branching ratio for $h_{f} \rightarrow \gamma \gamma$ would provide a very clear experimental signature, and observation of such a particle would strongly constrain the possible choices of the underlying Higgs sector [5]-[11].

Experimental searches for $h_{f}$ have been performed at the CERN Large Electron Positron Collider (LEP) and the Fermilab Tevatron. Lower limits on $m_{h_{f}}$ have been derived in the context of a benchmark model which assumes that the coupling $h_{f} V V$ is equal to the Standard Model (SM) Higgs boson coupling $\phi^{0} V V$, and that all fermion branching ratios are exactly zero. We will refer to this model as Benchmark F. Lower bounds

of the order $m_{h_{f}} \gtrsim 100 \mathrm{GeV}$ have been obtained by the LEP collaborations OPAL[12], DELPHI[13], ALEPH[14], and L3[15], utilizing the channel $e^{+} e^{-} \rightarrow h_{f} Z, h_{f} \rightarrow \gamma \gamma$. A search in the complementary channel $e^{+} e^{-} \rightarrow A^{0} h_{f}$ was performed by two LEP collaborations, OPAL [12] and DELPHI [13] and ruled out the region $m_{A}+m_{h_{f}}<160 \mathrm{GeV}$.

At the Tevatron Run I, the limits on $m_{h_{f}}$ from the D $\varnothing$ and CDF collaborations are respectively $78.5 \mathrm{GeV}$ [16] and $82 \mathrm{GeV}$ [17] at 95\% C.L., using the mechanism $q q^{\prime} \rightarrow V^{*} \rightarrow$ $h_{f} V, h_{f} \rightarrow \gamma \gamma$, with the dominant contribution coming from $V=W^{ \pm}$. For an integrated luminosity of $2 \mathrm{fb}^{-1}$ (which has been attained as of June 2007) Run II can extend the coverage of $m_{h_{f}}$ in Benchmark $F$ model slightly beyond that of LEP [18]-[20]. In addition, Run II is sensitive to the region $110 \mathrm{GeV}<m_{h_{f}}<160 \mathrm{GeV}$ and $B\left(h_{f} \rightarrow \gamma \gamma\right)>4 \%$ which could not be probed at LEP. A preliminary search in the inclusive $2 \gamma$ channel has been performed with $0.19 \mathrm{fb}^{-1}$ of Run II data [21, 22]. A complementary production mechanism which is exclusive to a hadron collider is the process $q q^{\prime} \rightarrow H^{ \pm} h_{f}$ [23, 24]. Since the branching ratio for $H^{ \pm} \rightarrow h_{f} W^{(*)}$ can be very large in models with fermiophobia [25] this mechanism can give rise to double $h_{f}$ production and hence a multiphoton signature. The sensitivity of the Tevatron Run II to this channel was studied in [26] and recently a search was performed by the $\mathrm{D} \varnothing$ collaboration in the $3 \gamma$ channel [27].

In Benchmark $F$ the decay $h_{f} \rightarrow \gamma \gamma$ is assumed to be mediated solely by $W^{ \pm}$loops. In this paper we study the effect of charged scalar loops $\left(H^{ \pm}\right)$on $B\left(h_{f} \rightarrow \gamma \gamma\right)$ in the context of the 2HDM (type I) and discuss the impact on the current and future searches for $h_{f}$ at the Tevatron and LHC. Our work is organized as follows: in section 2 the fermiophobic limit of the 2HDM (type I) is introduced; in section 3 the effect of the charged scalar loops on $h_{f} \rightarrow \gamma \gamma$ is discussed; the numerical results for $B\left(h_{f} \rightarrow \gamma \gamma\right)$ are contained in section 4 with conclusions given in section 5 . 


\section{Fermiophobic Higgs bosons}

In this section we briefly review the properties of $h_{f}$ in the 2HDM (type I). For a detailed introduction the reader is referred to [6], [9]-[11].

\section{$2.12 \mathrm{HDM}$ (Type I)}

If $\Phi_{1}$ and $\Phi_{2}$ are two Higgs $S U(2)$ doublets with hypercharge $Y=1$, the most general $S U(2)_{L} \times U(1)_{Y}$ gauge invariant scalar potential is [28]:

$$
\begin{aligned}
V= & m_{11}^{2} \Phi_{1}^{\dagger} \Phi_{1}+m_{22}^{2} \Phi_{2}^{\dagger} \Phi_{2}-\left(m_{12}^{2} \Phi_{1}^{\dagger} \Phi_{2}+\text { h.c. }\right)+\frac{1}{2} \lambda_{1}\left(\Phi_{1}^{\dagger} \Phi_{1}\right)^{2} \\
& +\frac{1}{2} \lambda_{2}\left(\Phi_{2}^{\dagger} \Phi_{2}\right)^{2}+\lambda_{3}\left(\Phi_{1}^{\dagger} \Phi_{1}\right)\left(\Phi_{2}^{\dagger} \Phi_{2}\right)+\lambda_{4}\left(\Phi_{1}^{\dagger} \Phi_{2}\right)\left(\Phi_{2}^{\dagger} \Phi_{1}\right) \\
& +\left\{\frac{1}{2} \lambda_{5}\left(\Phi_{1}^{\dagger} \Phi_{2}\right)^{2}+\left[\lambda_{6}\left(\Phi_{1}^{\dagger} \Phi_{1}\right)+\lambda_{7}\left(\Phi_{2}^{\dagger} \Phi_{2}\right)\right] \Phi_{1}^{\dagger} \Phi_{2}+\text { h.c. }\right\} .
\end{aligned}
$$

If the discrete symmetry $\Phi_{1} \rightarrow-\Phi_{1}$ is imposed one has $\lambda_{6}=\lambda_{7}=0$. However, the term proportional to $m_{12}^{2}$ can remain as a soft violation of the above discrete symmetry and still ensure that Higgs-mediated tree-level flavour changing neutral currents are absent [3]. Note that the above $2 \mathrm{HDM}$ potential contains one more free parameter than those studied in Refs. [10, 11]. We assume that all the scalar potential parameters are real.

The scalar potential in eq. (1) breaks $S U(2)_{L} \times U(1)_{Y}$ down to $U(1)_{e m}$ when the two Higgs doublets acquire vacuum expectation values

$$
\left\langle\Phi_{1}\right\rangle=\frac{1}{\sqrt{2}}\left(\begin{array}{c}
0 \\
v_{1}
\end{array}\right), \quad\left\langle\Phi_{2}\right\rangle=\frac{1}{\sqrt{2}}\left(\begin{array}{c}
0 \\
v_{2}
\end{array}\right)
$$

which must satisfy the experimental constraint $m_{Z}^{2}=\frac{1}{2}\left(g^{2}+g^{\prime 2}\right) v^{2}$, with $v^{2}=\left(v_{1}^{2}+v_{2}^{2}\right) \approx$ $(246 \mathrm{GeV})^{2}$. The minimization conditions that define the vacuum expectation values in terms of the parameters of the potential (setting $\lambda_{6}=\lambda_{7}=0$ ) are

$$
\begin{aligned}
& t_{1}=m_{11}^{2} v_{1}-m_{12}^{2} v_{2}+\frac{1}{2} \lambda_{1} v_{1}^{3}+\frac{1}{2}\left(\lambda_{3}+\lambda_{4}+\lambda_{5}\right) v_{1} v_{2}^{2}=0 \\
& t_{2}=m_{22}^{2} v_{2}-m_{12}^{2} v_{1}+\frac{1}{2} \lambda_{2} v_{2}^{3}+\frac{1}{2}\left(\lambda_{3}+\lambda_{4}+\lambda_{5}\right) v_{1}^{2} v_{2}=0
\end{aligned}
$$

from which $m_{11}^{2}$ and $m_{22}^{2}$ can be solved in favour of $m_{Z}^{2}$ and $\tan \beta \equiv v_{2} / v_{1}$.

After using the minimization conditions, the neutral CP-odd Higgs mass matrix can be written as

$$
\mathbf{M}_{A}^{2}=\left(\begin{array}{cc}
m_{12}^{2} t_{\beta}-\lambda_{5} v^{2} s_{\beta}^{2} & -m_{12}^{2}+\lambda_{5} v^{2} s_{\beta} c_{\beta} \\
-m_{12}^{2}+\lambda_{5} v^{2} s_{\beta} c_{\beta} & m_{12}^{2} / t_{\beta}-\lambda_{5} v^{2} c_{\beta}^{2}
\end{array}\right)
$$

and is diagonalized by a rotation in an angle $\beta$. For simplicity we use the following notation, $s_{\beta}=\sin \beta, c_{\beta}=\cos \beta$, and $t_{\beta}=\tan \beta$. The mass matrix $\mathbf{M}_{A}^{2}$ has a zero eigenvalue corresponding to the neutral Goldstone boson while its second eigenvalue is the mass of the physical CP-odd Higgs boson $A^{0}$,

$$
m_{A}^{2}=\frac{m_{12}^{2}}{s_{\beta} c_{\beta}}-\lambda_{5} v^{2}
$$


The charged Higgs mass matrix is given by

$$
\mathbf{M}_{H^{ \pm}}^{2}=\left(\begin{array}{cc}
m_{12}^{2} t_{\beta}-\frac{1}{2}\left(\lambda_{4}+\lambda_{5}\right) v^{2} s_{\beta}^{2} & -m_{12}^{2}+\frac{1}{2}\left(\lambda_{4}+\lambda_{5}\right) v^{2} s_{\beta} c_{\beta} \\
-m_{12}^{2}+\frac{1}{2}\left(\lambda_{4}+\lambda_{5}\right) v^{2} s_{\beta} c_{\beta} & m_{12}^{2} / t_{\beta}-\frac{1}{2}\left(\lambda_{4}+\lambda_{5}\right) v^{2} c_{\beta}^{2}
\end{array}\right)
$$

which also is diagonalized by a rotation in an angle $\beta$. It has a zero eigenvalue corresponding to the charged Goldstone boson, and the charged Higgs mass is

$$
m_{H^{ \pm}}^{2}=m_{A}^{2}+\frac{1}{2}\left(\lambda_{5}-\lambda_{4}\right) v^{2}
$$

Here we see that the charged and the CP-odd Higgs masses are independent parameters, as opposed to supersymmetry, where the mass squared difference is equal to $m_{W}^{2}$ at treelevel.

The neutral CP-even Higgs mass matrix is given by

$$
\mathbf{M}_{H^{0}}^{2}=\left(\begin{array}{cc}
m_{A}^{2} s_{\beta}^{2}+\lambda_{1} v^{2} c_{\beta}^{2}+\lambda_{5} v^{2} s_{\beta}^{2} & -m_{A}^{2} s_{\beta} c_{\beta}+\left(\lambda_{3}+\lambda_{4}\right) v^{2} s_{\beta} c_{\beta} \\
-m_{A}^{2} s_{\beta} c_{\beta}+\left(\lambda_{3}+\lambda_{4}\right) v^{2} s_{\beta} c_{\beta} & m_{A}^{2} c_{\beta}^{2}+\lambda_{2} v^{2} s_{\beta}^{2}+\lambda_{5} v^{2} c_{\beta}^{2}
\end{array}\right)
$$

and the two eigenvalues are the masses of the neutral CP-even Higgs bosons $h^{0}$ and $H^{0}$. It is diagonalized by an angle $\alpha$ defined by

$$
\sin 2 \alpha=\frac{\left[-m_{A}^{2}+\left(\lambda_{3}+\lambda_{4}\right) v^{2}\right] s_{2 \beta}}{\sqrt{\left[\left(m_{A}^{2}+\lambda_{5} v^{2}\right) c_{2 \beta}-\lambda_{1} v^{2} c_{\beta}^{2}+\lambda_{2} v^{2} s_{\beta}^{2}\right]^{2}+\left[m_{A}^{2}-\left(\lambda_{3}+\lambda_{4}\right) v^{2}\right]^{2} s_{2 \beta}^{2}}} .
$$

A necessary condition for fermiophobia is the imposition of the mentioned discrete symmetry $\Phi_{1} \rightarrow-\Phi_{1}$ which forbids $\Phi_{1}$ coupling to the fermions. This model is usually called "Type I" 2]. However, fermiophobia is only partial due to the mixing in the CPeven neutral Higgs mass matrix, which is diagonalized by the mixing angle $\alpha$, and both $\mathrm{CP}$-even mass eigenstates $h^{0}$ and $H^{0}$ acquire a coupling to the fermions. The fermionic couplings of the lightest CP-even Higgs $h^{0}$ take the form $h^{0} f \bar{f} \sim \cos \alpha / \sin \beta$, where $f$ is any fermion. Small values of $\cos \alpha$ would strongly suppress the fermionic couplings, and in the limit $\cos \alpha \rightarrow 0$ the coupling $h^{0} f \bar{f}$ would vanish, giving rise to complete fermiophobia at tree-level 1 From eq. (9) this is achieved if

$$
m_{A}^{2}=\left(\lambda_{3}+\lambda_{4}\right) v^{2} .
$$

Despite this extra constraint, the parameters $m_{A}, m_{H^{ \pm}}$, and $\tan \beta$ are still independent parameters in this model. However, at the one-loop level, $h_{f}$ can couple to fermions via loops involving vector bosons and other Higgs bosons (for a discussion see [6, 10, 11, 24]). Thus in general one would expect a small $B\left(h_{f} \rightarrow f \bar{f}\right)$ even if fermiophobia were exact at tree-level. Despite this, it is conventional and convenient to define an extreme $h_{f}$ in which all branching ratios to fermions are set to zero. This can be arranged by defining a vanishing renormalized $\alpha$.

\footnotetext{
${ }^{1}$ The limit $\sin \alpha \rightarrow 0$ is studied in Ref. 29] and can give rise to a very suppressed $B\left(h^{0} \rightarrow \gamma \gamma\right)$ in the 2HDM (Model I).
} 


\subsection{Fermiophobic Higgs boson branching ratios}

Expressions for the branching ratio $B\left(h_{f} \rightarrow \gamma \gamma\right)$ can be found in ref. [3]. In Benchmark $F$ this decay is assumed to be mediated solely by $W$ boson loops,
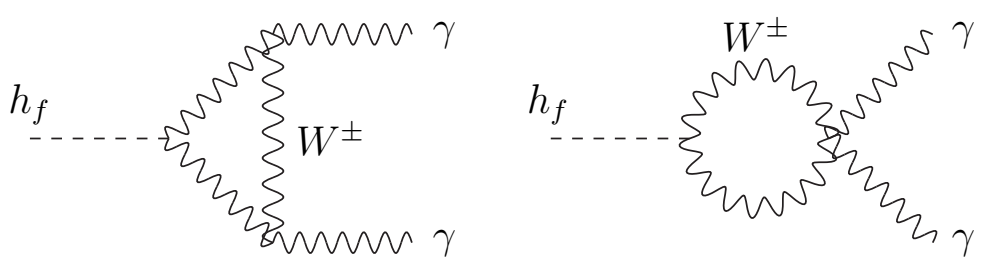

with a SM-strength Higgs- $W-W$ coupling, although in the 2 HDM a factor of $\sin (\beta-\alpha)$ must be included. In the fermiophobic limit $(\cos \alpha=0)$ the $h_{f} W W$ coupling $\left(g_{h_{f} W W}\right)$ normalized to the SM $\phi_{0} W W$ coupling satisfies $\sin (\beta-\alpha) \rightarrow-\cos \beta$. We call this scenario Benchmark $B$. The decay rate for $h_{f}$ into two photons is as follows:

$$
\Gamma\left(h_{f} \rightarrow \gamma \gamma\right)=\frac{\alpha^{2} g^{2}}{1024 \pi^{3}} \frac{m_{h_{f}}^{3}}{m_{W}^{2}}\left|F_{1} \cos \beta\right|^{2}
$$

Here $g$ is the $S U(2)_{L}$ coupling constant, $\alpha$ is the fine-structure constant, and $F_{1}=F_{1}(\tau)$ where $\tau=4 m_{W}^{2} / m_{h_{f}}^{2}$ is a phase space function given in [3].

For the sake of illustration, we depict in Fig. 1 the branching ratios of a fermiophobic Higgs boson $h_{f}$ into $V V$ where $V$ can be either a $W, Z$ or $\gamma$ (see [5, 6] for earlier versions of the same figure). In Fig. 1 it is assumed that all $h_{f}$ couplings to fermions are absent and that $h_{f} \rightarrow \gamma \gamma$ is mediated solely by a $W$ boson loop. One can see from the figure that the loop induced decay mode $h_{f} \rightarrow \gamma \gamma$ is dominant for $m_{h_{f}} \lesssim 95 \mathrm{GeV}$ and drops below $0.1 \%$ for $h_{f}$ masses above $150 \mathrm{GeV}$. On the other hand, the decay channel $h_{f} \rightarrow W^{*} W^{*}$ dominates for $m_{h_{f}} \gtrsim 95 \mathrm{GeV}$, being close to $100 \%$ until the threshold for $h_{f}$ decay into two real $Z$ 's is reached. Note that the branching ratios in Fig. 1 coincide for both Benchmark $B$ and $F$, although the decay rates and production cross-sections are different.

\section{Charged scalar loop contributions to $h_{f} \rightarrow \gamma \gamma$}

In the 2HDM (type I) there are additional contributions to $h_{f} \rightarrow \gamma \gamma$ from singly charged scalar $\left(H^{ \pm}\right)$loops $[3,10,11]^{2}$ whose graphs are shown below:

\footnotetext{
${ }^{2}$ See [30] for studies of the effect of charged scalar loops on the photonic decays of neutral Higgs bosons in the context of the $2 \mathrm{HDM}$ (type II).
} 


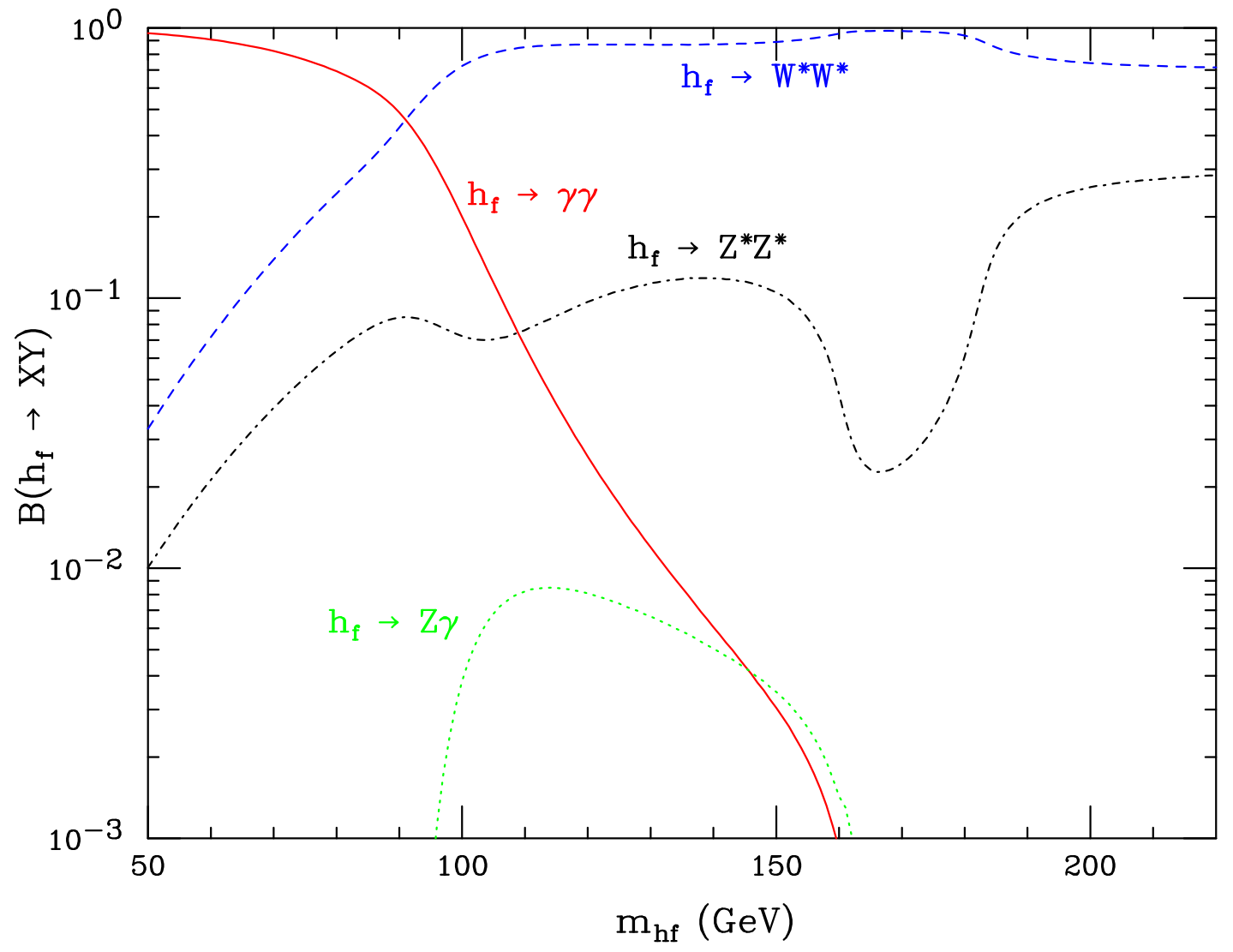

Figure 1: $B\left(h_{f} \rightarrow \gamma \gamma, W^{*} W^{*}, Z^{*} Z^{*}, Z \gamma\right)$ as a function of $m_{h_{f}}$ assuming all fermionic decays are absent (exact fermiophobic) in the Benchmark Models $F$ and $B$. 

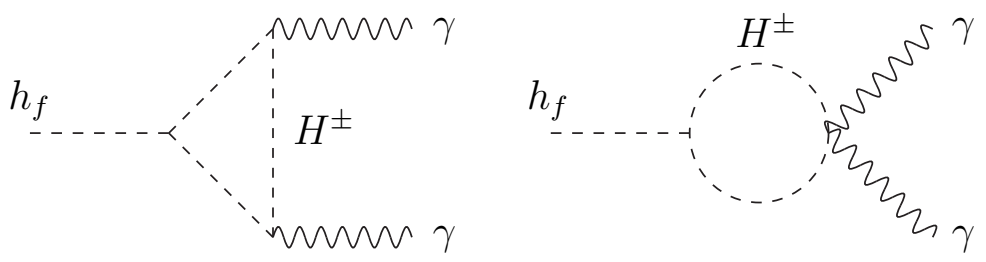

Such contributions, which are neglected in both Benchmark $B$ and $F$, introduce a dependence on the details of the Higgs spectrum. The expression for $\Gamma\left(h_{f} \rightarrow \gamma \gamma\right)$ in eq.(11) is modified to the following:

$$
\Gamma\left(h_{f} \rightarrow \gamma \gamma\right)=\frac{\alpha^{2} g^{2}}{1024 \pi^{3}} \frac{m_{h_{f}}^{3}}{m_{W}^{2}}\left|F_{0} \tilde{g}_{h_{f} H^{+} H^{-}}-F_{1} \cos \beta\right|^{2}
$$

Here the dimensionless coupling $\tilde{g}_{h_{f} H^{+} H^{-}}$is proportional to the trilinear coupling for the $h_{f} H^{+} H^{-}$vertex, which is defined below. $F_{0}$ is a phase space function of $\left(m_{H^{ \pm}}^{2} / m_{h_{f}}^{2}\right)$, i.e., the scalar analogue of $F_{1}$. In the mass region of interest to us, $100 \mathrm{GeV}<m_{h_{f}}<250 \mathrm{GeV}$, $F_{0}$ is considerably smaller than $F_{1}$ e.g., for $m_{h_{f}}<2 m_{H^{ \pm}}$one has $-1 / 3>F_{0}>-1$ while $7<F_{1}<12$ for $m_{h_{f}}<2 m_{W}$. However, the impact of the scalar loops can be significant if there is some suppression for the $h_{f} W W$ coupling or if $\tilde{g}_{h_{f} H^{+} H^{-}}$is sizeable. Hereafter we will refer to the scenario where the charged Higgs boson loops are included and the $2 \mathrm{HDM} g_{h_{f} W W}$ coupling is used as Benchmark $A$.

The trilinear coupling $\tilde{g}_{h_{f} H^{+} H^{-}}$in eq. (12) is related to the coupling in the Lagrangian, $L=g_{h_{f} H^{+} H^{-}} h_{f} H^{+} H^{-}+\ldots$, by

$$
\tilde{g}_{h_{f} H^{+} H^{-}}=-\frac{m_{W}}{g m_{H^{+}}^{2}} g_{h_{f} H^{+} H^{-}}
$$

where,

$$
g_{h_{f} H^{+} H^{-}}=c_{\beta} v\left[2 \frac{m_{H^{ \pm}}^{2}}{v^{2}}+s_{\beta}^{2} \lambda_{1}-\left(1+s_{\beta}^{2}\right) \frac{m_{A}^{2}}{v^{2}}-\left(1+s_{\beta}^{2}\right) \lambda_{5}\right]
$$

Eq. (14) is obtained by imposing the fermiophobic condition [eq. (10)] on the expression for $g_{h_{f} H^{+} H^{-}}$in the general 2HDM [28]. Note that at the Lagrangian level the trilinear coupling $g_{h_{f} H^{+} H^{-}}$has dimensions of mass.

Clearly the contribution of the $H^{ \pm}$loops depends on the details of the scalar potential. The phase space function $F_{0}$ involves the scalar masses $m_{h_{f}}$ and $m_{H^{ \pm}}$, while $g_{h_{f} H^{+} H^{-}}$is a function of several Higgs potential parameters. Since the charged scalar contribution may interfere destructively or constructively with that of the $W$ loop (depending on the sign of $g_{h_{f} H^{+} H^{-}}$), its main phenomenological effect on the decay $h_{f} \rightarrow \gamma \gamma$ is to increase or decrease $\mathrm{B}\left(h_{f} \rightarrow \gamma \gamma\right)$ for a given $m_{h_{f}}$ with respect to that given in Fig.1. A first study of the effect of the $H^{ \pm}$loops on $\mathrm{B}\left(h_{f} \rightarrow \gamma \gamma\right)$ in the fermiophobic limit of the 2HDM (type I) was performed in [10, 11]. The scalar potentials used in these references contain one less parameter than that given in eq. (1), and thus their corresponding expression for $g_{h_{f} H^{+} H^{-}}$ differs from that given in eq. (14). 
In this work we perform a general scan of the 2HDM parameter space in order to study the magnitude of $g_{h_{f} H^{+} H^{-}}$and the impact of the $H^{ \pm}$loops on $B\left(h_{f} \rightarrow \gamma \gamma\right)$. As input we use $\lambda_{i}(i=1,2,3,4,5)$ and $\tan \beta$. The mass parameters $m_{11}^{2}$ and $m_{22}^{2}$ in the potential are fixed by the minimization conditions in eq. (3). The third mass parameter $m_{12}^{2}$ is related to the CP-odd Higgs mass $m_{A}$, as shown in eq. (5), and fixed by the fermiophobic condition in eq. (10). The following perturbative limits for $\lambda_{i}$ are used:

$$
\begin{aligned}
0 & <\lambda_{1}, \lambda_{2}<4 \pi / 3 \\
-8 \pi & <2 \lambda_{3}, 2 \lambda_{4}, \lambda_{5}<8 \pi
\end{aligned}
$$

In addition, the vacuum stability conditions for $\lambda_{i}$ given in [28] are respected.

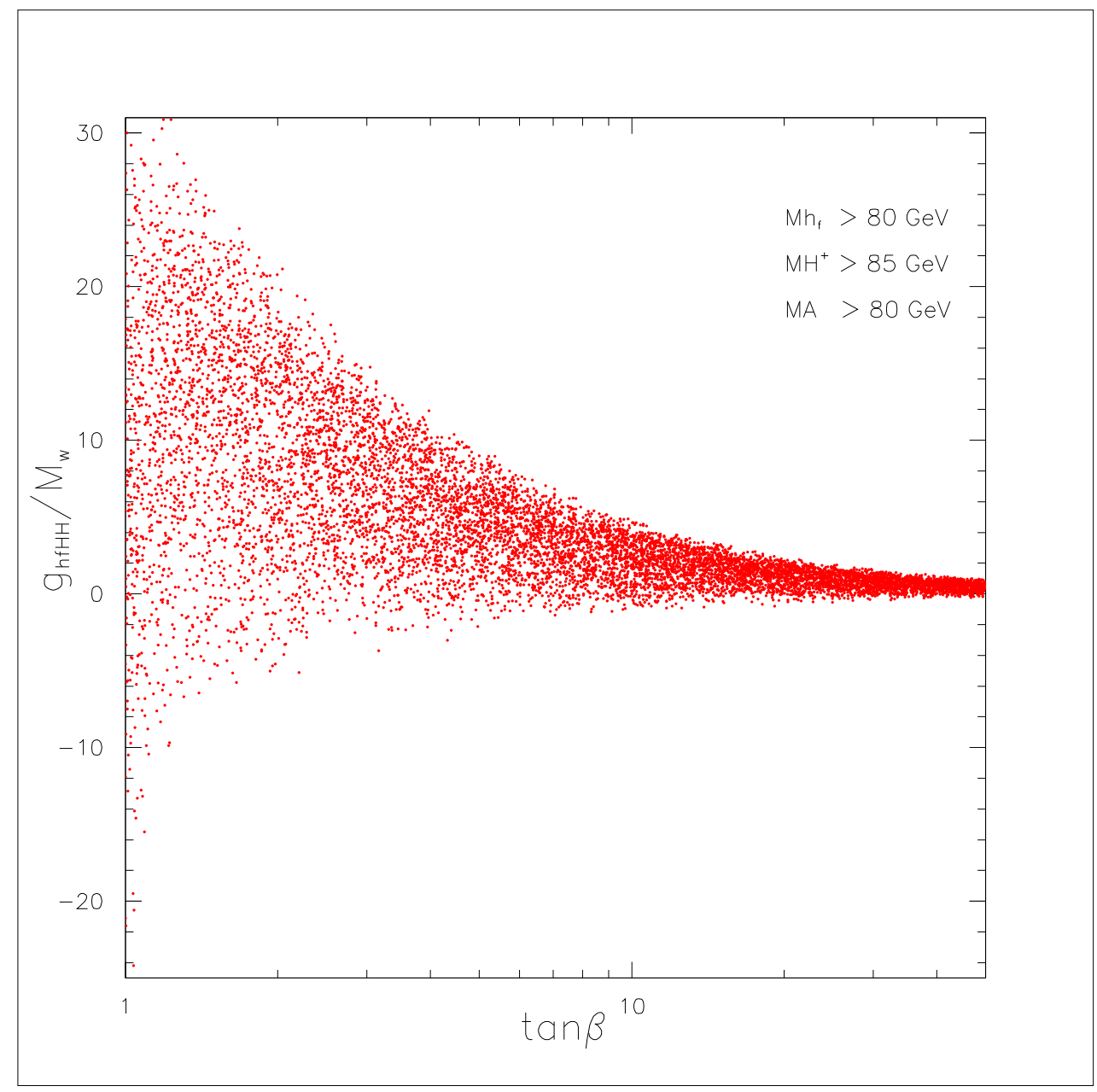

Figure 2: Fermiophobic 2HDM $h_{f} H^{+} H^{-}$coupling, normalized by $m_{W}$, as a function of $\tan \beta$.

In Fig. 2 the magnitude of the trilinear $g_{h_{f} H^{+} H^{-}}$coupling [eq. (14) normalized by $m_{W}$ ] is shown as a function of $\tan \beta$. We take $m_{h_{f}}, m_{A} \geq 80 \mathrm{GeV}$, and $m_{H^{+}} \geq 85 \mathrm{GeV}$ in order to comply with lower limits from direct searches. The $g_{h_{f} H^{+} H^{-}}$coupling may have either 
sign, with the largest values occurring for positive $g_{h_{f} H^{+} H^{-}}$. Most strikingly, the absolute value of the coupling diminishes with increasing $\tan \beta$, a fact that will have important consequences for the impact of the $H^{ \pm}$loops on $B\left(h_{f} \rightarrow \gamma \gamma\right)$.

\section{Numerical analysis}

In this section we perform a numerical analysis of the effect of charged scalar loops on $\mathrm{B}\left(h_{f} \rightarrow \gamma \gamma\right)$. We also study the impact on the current searches for $h_{f}$ at the Tevatron Run II and on future searches at the LHC. We consider both the standard production mechanism which depends on the $g_{h_{f} W W}$ coupling, and the complementary production mechanism which depends on the $g_{h_{f} H^{ \pm} W}$ coupling.

\subsection{Searches for $h_{f}$ at the Tevatron Run II}

Run II commenced in 2001 with the collision energy $\sqrt{s}$ increasing to $1.96 \mathrm{TeV}$. Simulations for the standard mechanism $p \bar{p} \rightarrow V \rightarrow V h_{f}\left(V=W^{ \pm}, Z\right)$ can be found in Refs. [18, 20]. It was shown that lower limits of $m_{h_{f}}>115(125) \mathrm{GeV}$ can be obtained for the Benchmark $F$ model with $2(10) \mathrm{fb}^{-1}$ of data, which is a moderate improvement over the LEP limits. However, the Tevatron search has the virtue of being sensitive to the parameter space of $110 \mathrm{GeV}<m_{h_{f}}<160 \mathrm{GeV}$, provided that $B\left(h_{f} \rightarrow \gamma \gamma\right)>4 \%$. In contrast, such a region could not be probed at LEP. Although these large branching ratios are not possible in the Benchmark $F$ model, we will discuss if contributions from charged scalar loops $\left(H^{ \pm}\right)$can provide the necessary enhancement.

A preliminary search for $p \bar{p} \rightarrow W^{*} \rightarrow W h_{f} \rightarrow \gamma \gamma+X$ has been carried out with a Run II data sample of $0.19 \mathrm{fb}^{-1}$ [21, 22]. Although the mass limit for $m_{h_{f}}$ in the Benchmark $F$ model is still inferior to that obtained at LEP, there is already sensitivity to the mass region $110 \mathrm{GeV}<m_{h_{f}}<160 \mathrm{GeV}$ and $B\left(h_{f} \rightarrow \gamma \gamma\right)>80 \%$. As of June 2007, $2 \mathrm{fb}^{-1}$ of data have been accumulated. In the fermiophobic limit in Benchmark $A$ this production mechanism is suppressed by a factor:

$$
g_{h_{f} W W}^{2} \sim \cos ^{2} \beta=\frac{1}{1+\tan ^{2} \beta}
$$

i.e., at $\tan \beta=3$ there is already a suppression factor $1 / 10$, and for this reason we will analyze this production mechanism at low values of $\tan \beta$. At larger values of $\tan \beta$ in Benchmark $A$ one must rely on complementary production mechanisms.

Recently a search (with $0.83 \mathrm{fb}^{-1}$ ) for a complementary process has been performed in the channel [27]:

$$
p \bar{p} \rightarrow h_{f} H^{ \pm} \rightarrow h_{f} h_{f} W^{ \pm} \rightarrow \gamma \gamma \gamma(\gamma)+X .
$$

Such a mechanism has very little SM background and the absence of a signal leads to the following limit on the production cross-section:

$$
\sigma\left(p \bar{p} \rightarrow h_{f} H^{ \pm}\right)<25.3 \mathrm{fb}
$$


Eq. (18) excludes regions in the parameter space of $m_{h_{f}}-m_{H^{ \pm}}$for a given $\tan \beta$ e.g. $m_{h_{f}}<44(50) \mathrm{GeV}$ and $m_{H^{ \pm}}<150 \mathrm{GeV}$ are excluded for $\tan \beta=3(30)$. All the above searches assume Benchmark $F$ model $B\left(h_{f} \rightarrow \gamma \gamma\right)$.

Prospects for $p p \rightarrow W^{*} \rightarrow W h_{f} \rightarrow \gamma \gamma+X$ at the LHC can be obtained from simulations for the SM Higgs boson production mechanism $p p \rightarrow W^{*} \rightarrow W \phi^{0} \rightarrow \gamma \gamma+X$ [31. For $m_{h_{f}}=120 \mathrm{GeV}$ in Benchmark $F$, a simple scaling of the signal rates in [31] gives statistical signals of $20 \sigma, 40 \sigma$ and $70 \sigma$ for $\mathcal{L}=30,100,300 \mathrm{fb}^{-1}$ respectively. For $m_{h_{f}}=150 \mathrm{GeV}, B\left(h_{f} \rightarrow \gamma \gamma\right)$ is approximately the same as $B\left(\phi^{0} \rightarrow \gamma \gamma\right)$ and a $5 \sigma$ signal can only be obtained with $\mathcal{L}=300 \mathrm{fb}^{-1}$. Simulations for the complementary channel in eq. (17) have not yet been performed for the LHC. Given the low backgrounds, one would expect considerably smaller values of $\sigma\left(p p \rightarrow h_{f} H^{ \pm}\right)$to be probed than the current upper limit set at the Tevatron Run II [eq. (18)]

\subsection{Impact of $H^{ \pm}$loops on $q q^{\prime} \rightarrow W^{*} \rightarrow W h_{f} \rightarrow \gamma \gamma+X$}

In Fig. 3 we show the fermiophobic Higgs boson Branching Ratio $B\left(h_{f} \rightarrow \gamma \gamma\right)$ for $\tan \beta=$ 1 and $m_{H^{ \pm}} \geq 115 \mathrm{GeV}$. The lower bound on $m_{H^{ \pm}}$ensures that the $H^{ \pm}$contribution to the decay $t \rightarrow H^{ \pm} b 32$ is consistent with the experimentally allowed regions. In the case of $b \rightarrow s \gamma$, the current world average is $B(b \rightarrow s \gamma)=(3.55 \pm 0.26) \times 10^{-4}$ [33]. Strong lower bounds on $m_{H^{ \pm}}$independent of $\tan \beta$ can be derived from this decay in the context of the 2HDM (type II), but in the 2HDM (type I) of interest to us such bounds are not applicable due to the different couplings of $H^{ \pm}$to fermions. For $\tan \beta>1$ the charged Higgs contribution is small, and the prediction for $B(b \rightarrow s \gamma)$ approaches that of the SM as $\tan \beta$ increases. For $\tan \beta=1$ the smaller values of $m_{H^{ \pm}}$used in this article predict $B(b \rightarrow s \gamma)$ somewhat lower than the experimentally allowed region, although this theoretical prediction is unstable for $\tan \beta \leq 1$ due to scale dependence [34]. In Fig. 3 Benchmark $F$ is denoted by the black points while Benchmark $A$ is denoted by green and red points for $\eta>1$ and $\eta<1$ respectively, with $\eta$ defined in the following way,

$$
\eta=\frac{\left.B\left(h_{f} \rightarrow \gamma \gamma\right)\right|_{A}}{\left.B\left(h_{f} \rightarrow \gamma \gamma\right)\right|_{F}}
$$

which is the ratio of the branching ratios in Benchmarks $A$ and $F$. In this way, for values of $\eta$ greater than unity, the charged Higgs and $W$ contributions add constructively. On the contrary, for values of $\eta$ smaller than unity, the two contributions add destructively.

A fermiophobic Higgs boson decaying into two photons was searched for at the Tevatron Run II with $0.19 \mathrm{fb}^{-1}$ [22]. The excluded region in the parameter space $\sigma(p \bar{p} \rightarrow$ $\left.h_{f} W\right) B\left(h_{f} \rightarrow \gamma \gamma\right) \mathrm{v} / \mathrm{s} m_{h_{f}}$ (Fig. 2 in [22]) is marginally increased with respect to LEP. However, a Montecarlo prediction for $2 \mathrm{fb}^{-1}$ shows a significant improvement over LEP. Assuming Benchmark F, i.e., no suppression factor in the cross section, and no charged Higgs contribution to the branching ratio, a fermiophobic Higgs mass $m_{h_{f}} \lesssim 113 \mathrm{GeV}$ would be probed. However, in Benchmark $A$ one has to include the suppression factor in the production cross section (eq. 16) and the contribution of the charged Higgs boson to the Higgs decay rate into two photons. In Fig. 3 we use $\tan \beta=1$ which gives rise to 
a suppression factor $g_{h_{f} W W}^{2}=1 / 2$. A few scenarios in 2HDM where the charged Higgs contribution to $B\left(h_{f} \rightarrow \gamma \gamma\right)$ overcomes the suppression factor (i.e., $\eta>2$ ) are observed in Fig. 3 for large values of $m_{h_{f}}$ and small values of $m_{H^{ \pm}}$. However, these large values $m_{h_{f}} \sim 250 \mathrm{GeV}$ together with the small $B\left(h_{f} \rightarrow \gamma \gamma\right) \sim 10^{-4}$ are well beyond the sensitivity of the Tevatron Run II.

In the mass region sensitive to Run II, $m_{h_{f}} \lesssim 160 \mathrm{GeV}$, we find $\eta \lesssim 1.1$ and thus the constructive effects of the charged Higgs contribution to $B\left(h_{f} \rightarrow \gamma \gamma\right)$ are far from compensating the suppression factor. Hence the use of Benchmark $F$ is reasonable for Run II when the search is negative and only lower bounds on the fermiophobic Higgs mass are set. We conclude that searches in the channel $q q^{\prime} \rightarrow W^{*} \rightarrow W h_{f} \rightarrow \gamma \gamma+X$ with the anticipated Run II luminosity of a few $\mathrm{fb}^{-1}$ offer similar sensitivity to Benchmark $A$ as the LEP searches.

At the LHC prospects are much brighter since statistically significant signals would be expected in Benchmark $F$ in the region $120 \mathrm{GeV}<m_{h_{f}}<150 \mathrm{GeV}$ (see Section 4.1). If a signal were observed in the above mass region, interpretation in the 2HDM (type I) (i.e. Benchmark A) would require inclusion of the scalar loops, whose effect on $B\left(h_{f} \rightarrow \gamma \gamma\right)$ can be sizeable as shown in Fig. 3. In the case of destructive interference for $m_{h_{f}} \sim 150$ $\mathrm{GeV}$ their contribution can be as large as $-90 \%(\eta \geq 0.1)$; in the constructive case, their contribution has an upper limit of the order of $10 \%(\eta \leq 1.1)$ for the same value of $m_{h_{f}}$. The signal event number in Benchmark $A$ would be proportional to the cross section suppression factor [eq. (16)] and $\eta$. Information on the magnitude of $\eta$ would restrict the parameter space of the Higgs potential via eq. (12) and eq. (14). Since Benchmark F can give very large signals for lighter values of $m_{h_{f}}$ (e.g. $70 \sigma$ for $m_{h_{f}}=120 \mathrm{GeV}$ and 300 $\mathrm{fb}^{-1}$ ), even small values of $\eta$ could be probed in Benchmark $A$ for $m_{h_{f}} \sim 120 \mathrm{GeV}$.

Note that the charged Higgs contribution to $B\left(h_{f} \rightarrow \gamma \gamma\right)$ rises sharply near the threshold $h_{f} \rightarrow H^{+} H^{-}$. Since $m_{H^{ \pm}}>115 \mathrm{GeV}$ in our example, the threshold starts appearing at $230 \mathrm{GeV}$. It is clear from Fig. 2c that the maximal value for $\eta$ is obtained for light charged Higgs bosons.

\subsection{Impact of $H^{ \pm}$loops on $q q^{\prime} \rightarrow H^{ \pm} h_{f} \rightarrow \gamma \gamma \gamma \gamma+X$}

Complementary mechanisms play an important role in the search for $h_{f}$ in the case of the $g_{h_{f} V V}$ coupling being suppressed, i.e., for large $\tan \beta$. For this reason we will consider the process $p p, p \bar{p} \rightarrow H^{ \pm} h_{f} \rightarrow \gamma \gamma \gamma \gamma+X$ [eq. (17)]. The total cross section is given by

$$
\sigma\left(p p, p \bar{p} \rightarrow H^{ \pm} h_{f}\right) \times B\left(H^{ \pm} \rightarrow W h_{f}\right) \times B^{2}\left(h_{f} \rightarrow \gamma \gamma\right)
$$

The inclusion of the charged Higgs loops will affect the total cross section quadratically, as can be seen in eq.(20), and thus their effect might be more important than for the standard mechanism.

In Fig 4 we show $B\left(h_{f} \rightarrow \gamma \gamma\right)$ as a function of $m_{h_{f}}$ for $\tan \beta=30$. Since there is no cross section suppression factor analogous to eq. (16), the signal event rate in Benchmark $A$ will be enhanced relative to that in Benchmark $F$ for $\eta>1$. In our scan of the $2 \mathrm{HDM}$ parameter space we impose a weaker lower limit for the charged Higgs mass $\left(m_{H^{ \pm}}>85\right.$ 
$\mathrm{GeV}$ ) than in Section 4.2. This is because the rate for $t \rightarrow H^{ \pm} b$ and the $H^{ \pm}$contribution to $b \rightarrow s \gamma$ are negligible for $\tan \beta=30$ in the $2 \mathrm{HDM}$ (type I). We plot both the constructive (green dots) and destructive (red dots) contribution to the fermiophobic Higgs decay to two photons. In this figure, as in Fig. 3, one sees a rise in the contribution to $B\left(h_{f} \rightarrow \gamma \gamma\right)$ (both constructive and destructive) close to the charged Higgs threshold. In this case $m_{H^{ \pm}}>85 \mathrm{GeV}$ and so the threshold starts to appear at $170 \mathrm{GeV}$. In the mass region sensitive to the LHC, corrections from charged Higgs bosons are very large; at $m_{h_{f}} \sim 150$ $\mathrm{GeV}$ they can reach $25 \%$ in the constructive case $(\eta \leq 1.25)$, and $-99 \%$ in the destructive case $(\eta \geq 0.01)$.

Since the $W$ contribution to $B\left(h_{f} \rightarrow \gamma \gamma\right)$ is proportional to $\cos \beta$ [eq. (12)], one might expect the influence of the charged Higgs contribution to be greater at large $\tan \beta$ when the $h_{f} W W$ coupling is very suppressed. However, from eq. (14) one sees that in the fermiophobic limit, the $h_{f} H^{+} H^{-}$coupling is also suppressed by a factor $\cos \beta$. For this reason the effect of the charged scalar loops is comparable in both Fig. 4 and Fig. 3 , contrary to the naïve expectation.

The lowest values of $\eta$ seen in Fig. 4 can be understood as a cancellation between the $W$ and $H^{ \pm}$amplitudes contributing to the $h_{f} \rightarrow \gamma \gamma$ decay width, which in the notation of eq. (12) translates into $F_{0} \tilde{g}_{h_{f} H^{+} H^{-}}=F_{1} \cos \beta$ for an exact cancellation. For the case with $m_{h_{f}} \approx 140 \mathrm{GeV}$ and $\eta \approx 10^{-2}$, best seen in Fig. $4 \mathrm{~b}$, both amplitudes are real and have opposite signs, but the value of $\eta$ cannot be lowered significantly because the parameter $\lambda_{1}$ would exceed its perturbative limit shown in eq. (15). For the case with $m_{h_{f}} \approx 220$ $\mathrm{GeV}$ and $\eta \approx 10^{-3}$, both amplitudes have imaginary parts, since for this case $m_{H^{+}} \approx 103$ $\mathrm{GeV}$, and can be produced on-shell in the decay of $h_{f}$. To obtain an exact cancellation between the $W$ and $H^{ \pm}$amplitudes in this case, it is necessary that the ratio of the real parts of $F_{1}$ and $F_{0}$ be equal to the ratio of their imaginary parts. It can be shown that this is not possible to achieve, and that is the reason why we do not find smaller values of $\eta$.

In Fig. 5 we show different regions in the plane formed by the $h_{f} H^{+} H^{-}$coupling $g_{h_{f} H^{+} H^{-}}$, normalized by $m_{W}$, and the charged Higgs mass $m_{H^{ \pm}}$, for two values of $\tan \beta=$ 1,30. The regions are defined by the parameter $\eta$ in the following way: I) for a given value of $m_{h_{f}}, B\left(h_{f} \rightarrow \gamma \gamma\right)$ calculated in Benchmark $A$ is within $2 \%$ of the branching ratio calculated in Benchmark F; II) the deviation is between $2 \%$ and $10 \%$; III) the deviation is larger than $10 \%$. The dividing lines are not absolute, since there is some small overlap between regions. As expected, larger corrections are obtained for larger values of the coupling $g_{h_{f} H^{+} H^{-}}$. Similarly, larger corrections are obtained for smaller charged Higgs masses. This behaviour can be understood from eq. (13), where we see that for fixed $g_{h_{f} H^{+} H^{-}}, \tilde{g}_{h_{f} H^{+} H^{-}}$is inversely proportional to $m_{H^{ \pm}}^{2}$. The effect of $\tan \beta$ can also be observed from the figure. The general shape of the regions is unchanged, and the observable effect is a re-scaling of the coupling $g_{h_{f} H^{+} H^{-}}$. This is clearly understood from eq. (14), where we see that both couplings $g_{h_{f} H^{+} H^{-}}$and $g_{h_{f} W^{+} W^{-}}$are scaled by a factor $\cos \beta$.

Note that the sign of the coupling $g_{h_{f} H^{+} H^{-}}$directly determines whether the charged Higgs contribution adds constructively $(\eta>1)$ or destructively $(\eta<1)$ to the $W$ contri- 
bution. In eq. (14) this coupling is written as a function of $m_{H^{ \pm}}, m_{A}, \lambda_{1}, \lambda_{5}$, and $\tan \beta$. The charged Higgs mass $m_{H^{ \pm}}$and $\lambda_{1}$ always contribute positively to $g_{h_{f} H^{+} H^{-}}$. On the contrary, the CP-odd Higgs mass $m_{A}$ contributes negatively, while the $\lambda_{5}$ contribution does not have a definite sign. As stressed in Section 4.2, observation of $h_{f} \rightarrow \gamma \gamma$ at the LHC with a sizeable event number would provide information on the magnitude of $\eta$, which in turn would constrain the above Higgs potential parameters in the context of the 2HDM (type I).

At the LHC, with $100 \mathrm{fb}^{-1}$ per experiment, the expected accuracy for the branching ratio $B\left(\phi_{0} \rightarrow \gamma \gamma\right)$ varies between $14 \%$ and $22 \%$, within the mass region sensitive to the LHC, $115<m_{\phi_{0}}<150 \mathrm{GeV}$ [35]. Considering that this study was done based on the $g g \rightarrow \phi_{0}$ production mechanism, it does not directly apply to our fermiophobic Higgs. This is because the relevant production mechanisms for $h_{f}$ at the LHC are higgsstrahlung and weak vector boson fusion, and a dedicated study would be needed. Nevertheless, it is encouraging that the corrections we find are easily larger than the quoted sensitivity. Even better precision for $B\left(\phi_{0} \rightarrow \gamma \gamma\right)$ can be achieved at the ILC, being $16 \%$ for $\sqrt{s}=500$ $\mathrm{GeV}$, and improvable to $10 \%$ with initial state polarization [36], with higgsstrahlung $e^{+} e^{-} \rightarrow Z \phi_{0}$ production mechanism, i.e., applicable to a fermiophobic Higgs. Finally, the photon-photon option for the ILC can achieve a $5 \%$ and $8 \%$ sensitivity for the modes $\gamma \gamma \rightarrow \phi_{0} \rightarrow W^{+} W^{-}$and $\gamma \gamma \rightarrow \phi_{0} \rightarrow \gamma \gamma$, respectively [37], which are also applicable to our fermiophobic Higgs $h_{f}$.

\section{Conclusions}

The LHC has impressive sensitivity to fermiophobic Higgs bosons $\left(h_{f}\right)$ decaying to two photons with a large $(>1 \%)$ branching ratio. Observation of this photonic decay mode $\left(h_{f} \rightarrow \gamma \gamma\right)$ with a rate significantly above that expected for the Standard Model Higgs boson could be accommodated in the fermiophobic limit of the 2HDM (type I). In a commonly used benchmark model the decay $h_{f} \rightarrow \gamma \gamma$ is assumed to be mediated solely by loops involving $W^{ \pm}$, although potentially large contributions may arise from charged

scalar loops. In the mass region sensitive to the LHC $\left(m_{h_{f}}<150 \mathrm{GeV}\right)$ we showed that such contributions can cause large suppressions, $\eta \geq 0.01$ ( $-99 \%$ correction) or moderate enhancements, $\eta \leq 1.25$ (25\% correction) of the branching ratio for $h_{f} \rightarrow \gamma \gamma$. These corrections should be compared with expected sensitivities in the measurement of the photon-photon branching ratio of the Higgs, which vary from $22 \%$ at the LHC to $5 \%$ at the photon-photon option of the ILC. Consequently, interpretation in the 2HDM (type I) of any signal for $h_{f}$ at the LHC and ILC would necessitate inclusion of the scalar loops. This in turn would provide information on the parameters of the scalar potential, through the charged Higgs mass and the $h_{f} H^{+} H^{-}$coupling, which in the fermiophobic limit was shown to diminish with increasing $\tan \beta$, as does $h_{f} W^{+} W^{-}$. 


\section{Acknowledgments}

A.G.A. was supported by National Cheng Kung University Grant No. OUA 95-3-2-057. M.A.D. was supported by Anillo "Centro de Estudios Subatomicos" grant. M.A.R. is thankful to Conicyt for their support. A.G.A. is grateful for travel support from Fondecyt project No. 7060202 and for hospitality at La Universidad Católica de Chile where this work was initiated.

\section{References}

[1] T. J. Weiler, Proceedings of the 8th Vanderbilt Int. Conf. on High Energy Physics, Nashville, TN, Oct 8-10, 198\%; Edited by J. Brau and R. Panvini (World Scientific, Singapore, 1988), p219

[2] H. E. Haber, G. L. Kane and T. Sterling, Nucl. Phys. B 161, 493 (1979).

[3] J. F. Gunion, H. E. Haber, G. L. Kane and S. Dawson, "The Higgs Hunter's Guide," (Reading, MA: Addison-Wesley, 1989)

[4] H. Georgi and M. Machacek, Nucl. Phys. B 262, 463 (1985); M. S. Chanowitz and M. Golden, Phys. Lett. B 165, 105 (1985).

[5] A. Stange, W. J. Marciano and S. Willenbrock, Phys. Rev. D 49, 1354 (1994).

[6] M. A. Diaz and T. J. Weiler, arXiv:hep-ph/9401259.

[7] V. D. Barger, N. G. Deshpande, J. L. Hewett and T. G. Rizzo, arXiv:hep-ph/9211234. In Argonne 1993, Physics at current accelerators and supercolliders* 437-442

[8] H. Pois, T. J. Weiler and T. C. Yuan, Phys. Rev. D 47, 3886 (1993).

[9] A. G. Akeroyd, Phys. Lett. B 368, 89 (1996).

[10] A. Barroso, L. Brucher and R. Santos, Phys. Rev. D 60, 035005 (1999).

[11] L. Brucher and R. Santos, Eur. Phys. J. C 12, 87 (2000).

[12] G. Abbiendi et al. [OPAL Collaboration], Phys. Lett. B 544, 44 (2002).

[13] P. Abreu et al. [DELPHI Collaboration], Phys. Lett. B 507, 89 (2001); Eur. Phys. J. C 35, 313 (2004).

[14] A. Heister et al. [ALEPH Collaboration], Phys. Lett. B 544, 16 (2002).

[15] P. Achard et al. [L3 Collaboration], Phys. Lett. B 534, 28 (2002); Phys. Lett. B 568, 191 (2003).

[16] B. Abbott et al. [DØ Collaboration], Phys. Rev. Lett. 82, 2244 (1999). 
[17] T. Affolder et al. [CDF Collaboration], Phys. Rev. D 64, 092002 (2001).

[18] S. Mrenna and J. Wells, Phys. Rev. D 63, 015006 (2001).

[19] V. M. Abazov et al. [D0 Collaboration], arXiv:hep-ex/0508054.

[20] G. Landsberg and K. T. Matchev, Phys. Rev. D 62, 035004 (2000).

[21] A. Melnitchouk [D0 Collaboration], Int. J. Mod. Phys. A 20, 3305 (2005).

[22] http://www-d0.fnal.gov/Run2Physics/WWW/results/higgs.htm, D0 Note 4374CONF.

[23] A. G. Akeroyd and M. A. Diaz, Phys. Rev. D 67, 095007 (2003).

[24] A. G. Akeroyd, M. A. Diaz and F. J. Pacheco, Phys. Rev. D 70, 075002 (2004).

[25] A. G. Akeroyd, Nucl. Phys. B 544, 557 (1999).

[26] A. G. Akeroyd, A. Alves, M. A. Diaz and O. J. P. Eboli, Eur. Phys. J. C 48, 147 (2006).

[27] G. L. Landsberg [CDF Collaborations], arXiv:0705.2855 [hep-ex]; http://www-d0.fnal.gov/Run2Physics/WWW/results/higgs.htm, D0 Note 5067CONF.

[28] J. F. Gunion and H. E. Haber, Phys. Rev. D 67, 075019 (2003).

[29] D. Phalen, B. Thomas and J. D. Wells, Phys. Rev. D 75, 117702 (2007).

[30] I. F. Ginzburg, M. Krawczyk and P. Osland, Nucl. Instrum. Meth. A 472, 149 (2001); A. Arhrib, W. Hollik, S. Penaranda and M. Capdequi Peyranere, Phys. Lett. B 579, 361 (2004).

[31] M. Lethuillier, O. Ravat, J. L. Agram, C. Baty, S. Gascon-Shotkin and S. Perries, J. Phys. G 34 (2007) N105.

[32] A. Abulencia et al. [CDF Collaboration], Phys. Rev. Lett. 96, 042003 (2006).

[33] E. Barberio et al. [Heavy Flavor Averaging Group (HFAG)], arXiv:hep-ex/0603003.

[34] F. Borzumati and C. Greub, Phys. Rev. D 58, 074004 (1998); F. Borzumati and C. Greub, Phys. Rev. D 59, 057501 (1999).

[35] D. Zeppenfeld, in Proc. of the APS/DPF/DPB Summer Study on the Future of Particle Physics (Snowmass 2001) ed. N. Graf, In the Proceedings of APS / DPF / DPB Summer Study on the Future of Particle Physics (Snowmass 2001), Snowmass, Colorado, 30 Jun - 21 Jul 2001, pp P123 arXiv:hep-ph/0203123. 
[36] E. Boos, J. C. Brient, D. W. Reid, H. J. Schreiber and R. Shanidze, Eur. Phys. J. C 19, 455 (2001).

[37] D. Asner et al., Eur. Phys. J. C 28, 27 (2003). 


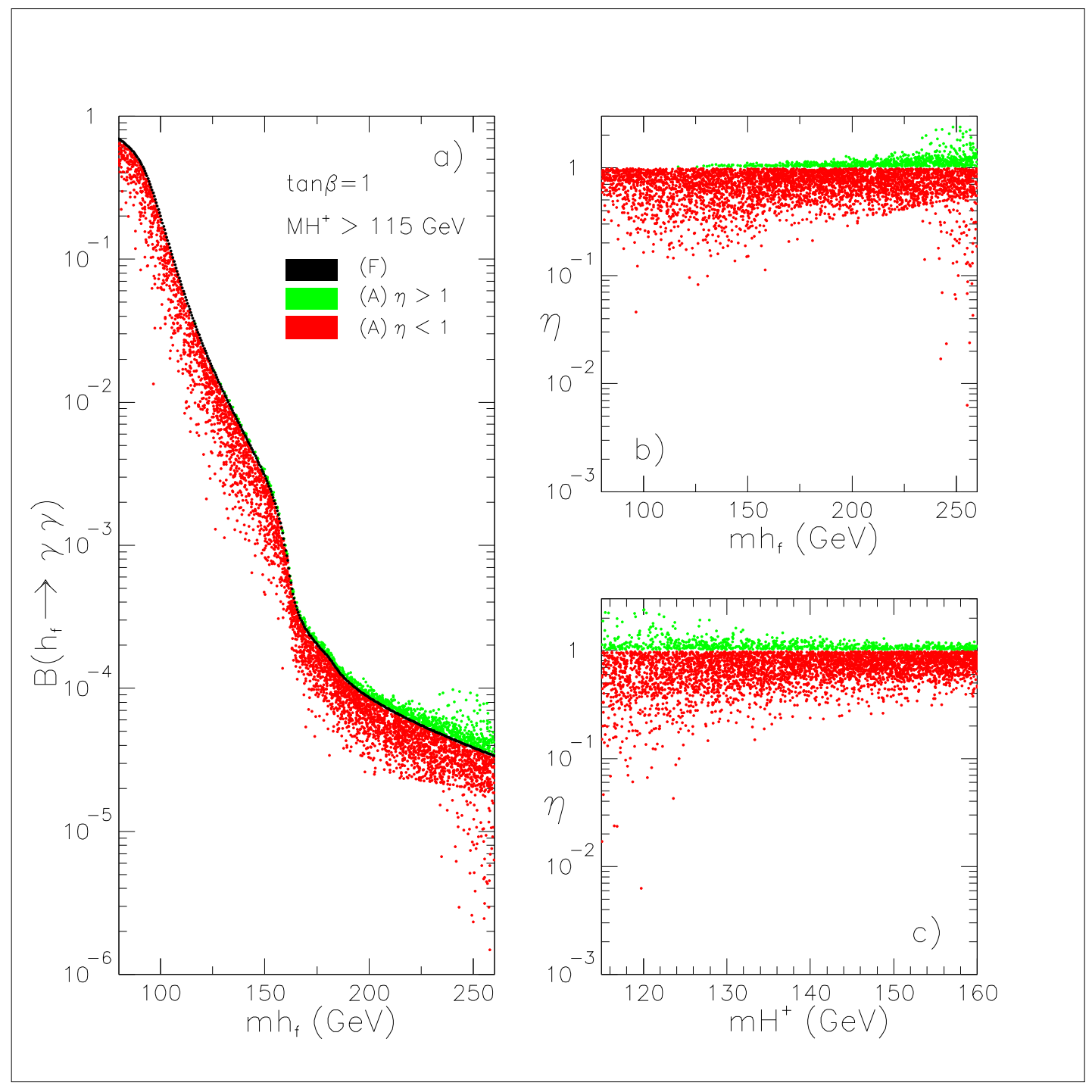

Figure 3: For $\tan \beta=1$ the three panels are as follows: a) $B\left(h_{f} \rightarrow \gamma \gamma\right)$ in Benchmark $F$ (black dots), and in Benchmark $A$ with $\eta>1$ (green dots) and $\eta<1$ (red dots). b) Scatter plot for $\eta$ as a function of the fermiophobic Higgs mass $m_{h_{f}}$. c) Scatter plot for $\eta$ as a function of the charged Higgs mass $m_{H^{ \pm}}$. 


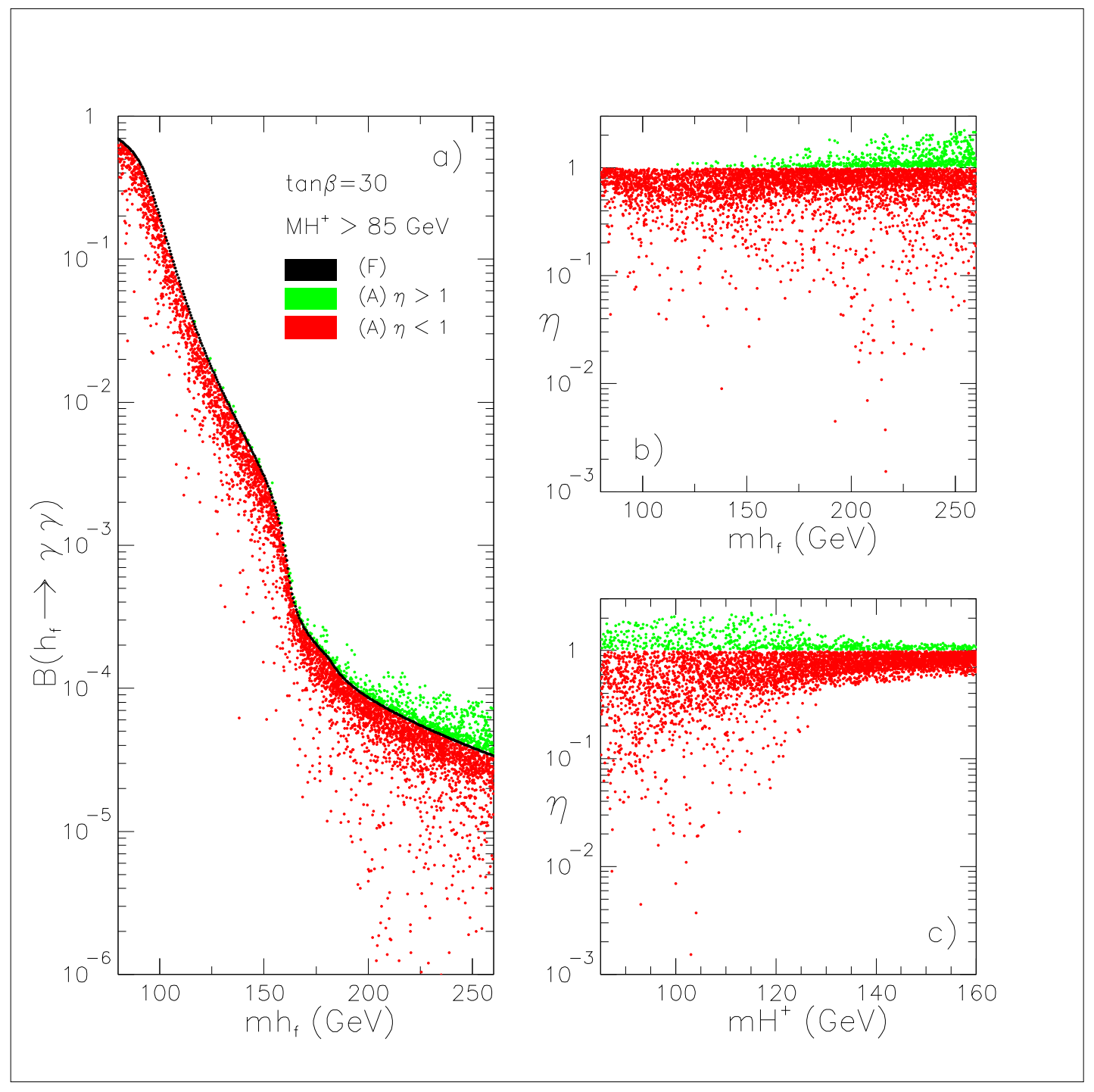

Figure 4: For $\tan \beta=30$ the three panels are as follows: a) $B\left(h_{f} \rightarrow \gamma \gamma\right)$ in Benchmark $F$ (black dots), and in Benchmark $A$ with $\eta>1$ (green dots) and $\eta<1$ (red dots). b) Scatter plot for $\eta$ as a function of the fermiophobic Higgs mass $m_{h_{f}}$. c) Scatter plot for $\eta$ as a function of the charged Higgs mass $m_{H^{ \pm}}$. 

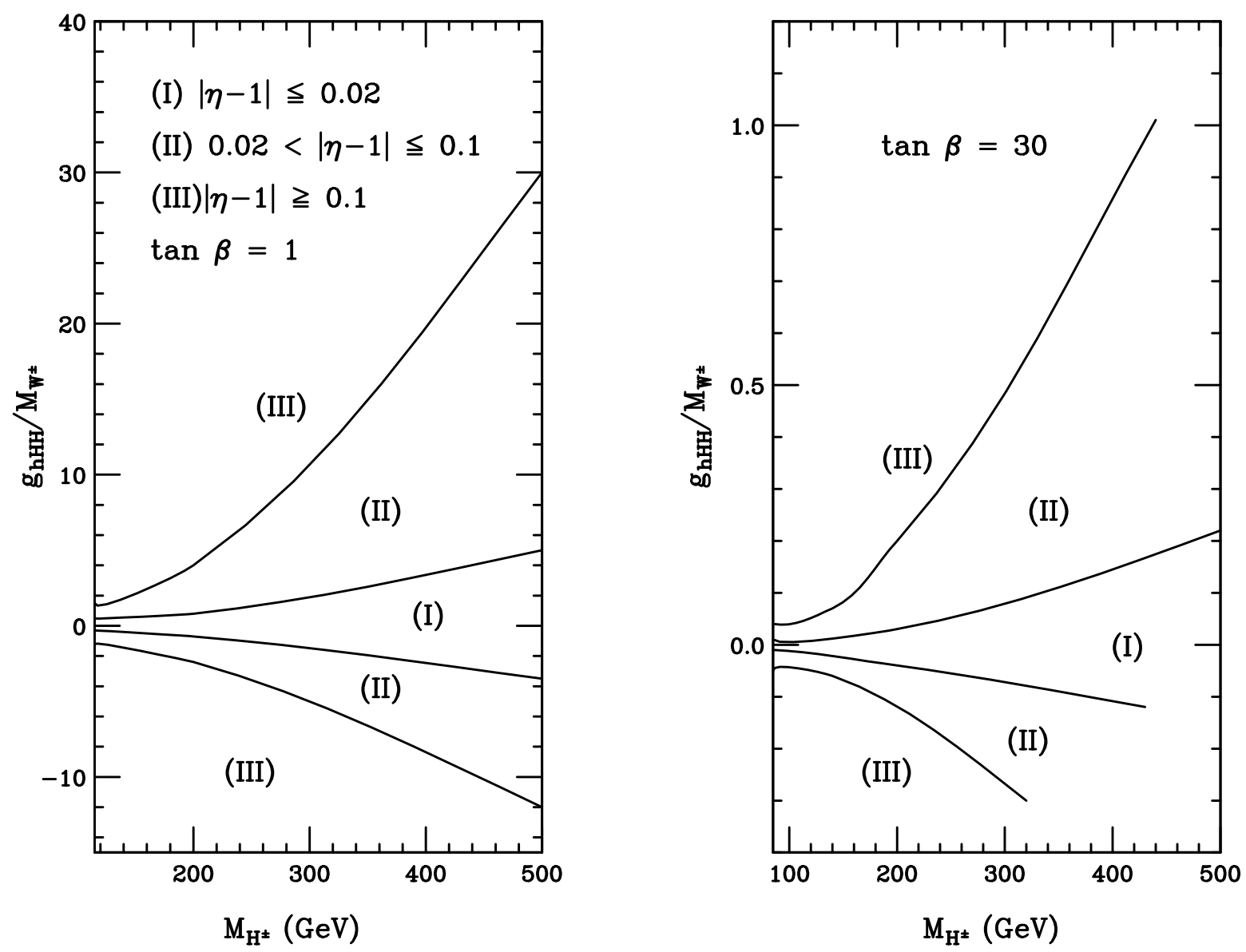

Figure 5: In the plane of parameter space defined by the charged Higgs mass $m_{H^{ \pm}}$and the $h_{f} H^{+} H^{-}$coupling $g_{h_{f} H^{+} H^{-}}$(normalized by $m_{W}$ ), three different regions are displayed: I) Charged Higgs corrections within $2 \%$, II) between $2 \%$ and $10 \%$, and III) larger than $10 \%$, for two values of $\tan \beta=1,30$. 